\title{
ANHANG
}

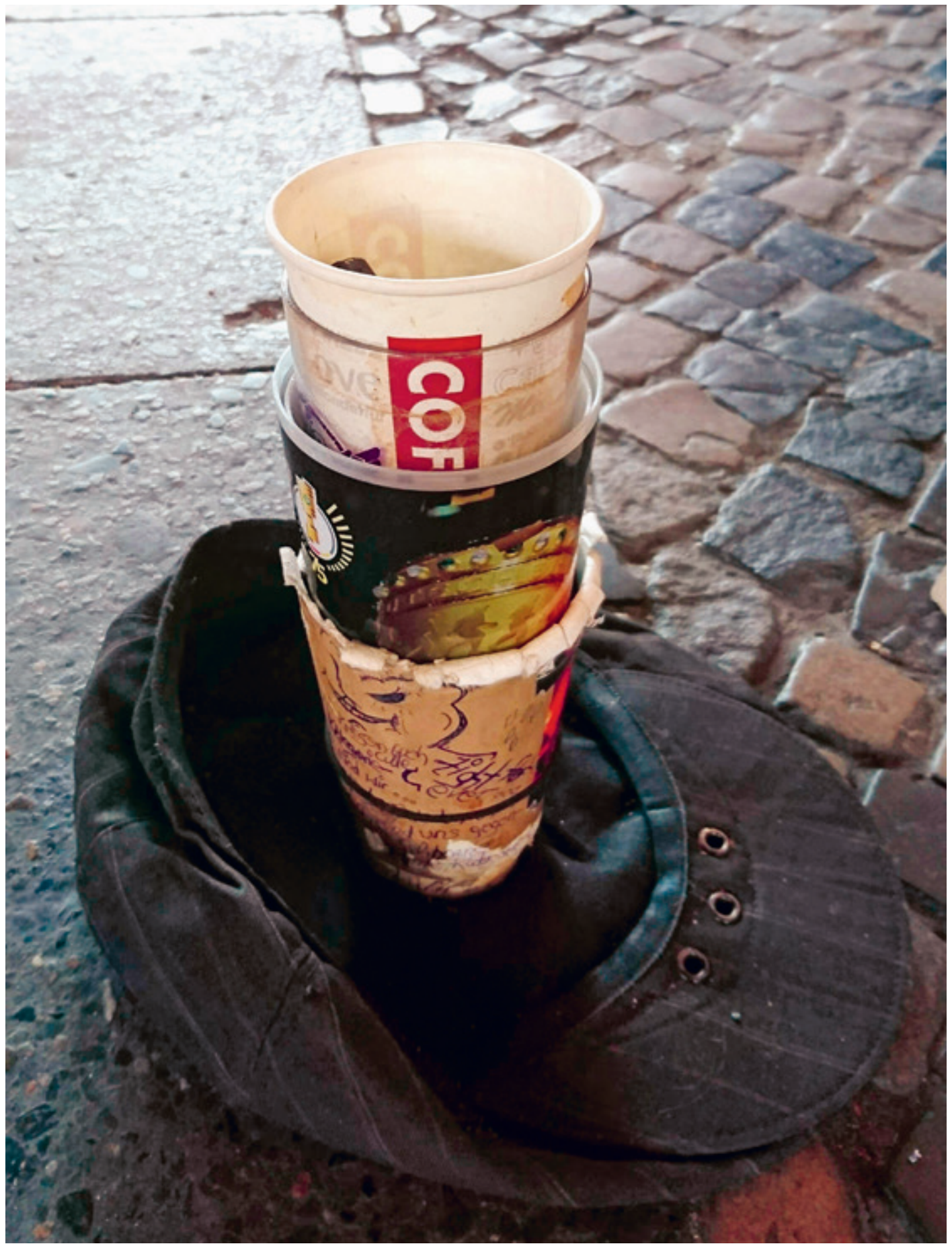


ist Künstler und Stadtforscher, lebt und arbeitet in Zürich und Hannover. Seit 2001 ist er an der Zürcher Hochschule der Künste (ZHdK), dort seit 2011 am Institut für Gegenwartskunst (IFCAR) tätig. Seine Forschungs-, Publikations- und Lehrtätigkeiten siedeln sich im Bereich der künstlerischethnografischen Stadtforschung an. Ein besonderer Fokus liegt hierbei auf dem Thema der „Offenen Stadt“. Weitere Arbeitsfelder sind bildbasierte Forschungsmethoden sowie der Vergleich zwischen ostasiatischen und westlichen Stadt- und Raumkonzepten. Krusche arbeitete unter anderem in Tokyo und Shanghai, Berlin und Belgrad, zuletzt an einem Projekt in Hongkong (www.shamshuipo-deepwater.com).

Jüngste Publikationen:

Krusche, Jürgen/King Chung, Siu (Hg.) (2018): Deep Water. Public Spaces in Sham Shui Po, Hong Kong, MCCM Creations, Hong Kong, https://www.mccmcreations.com/product-page/deep-water Krusche, Jürgen (Hg.) (2017): Die Ambivalente Stadt. Gegenwart und Zukunft des öffentlichen Raums, JOVIS Verlag, Berlin, www.jovis.de/de/buecher/details/product/die_ambivalente_stadt.html

\section{Aya Domenig}

studierte an der Universität Zürich visuelle Anthropologie, Filmwissenschaft und Japanologie. Anschließend absolvierte sie an der Zürcher Hochschule der Künste (ZHdK) ein Studium der Filmregie. Ihr Diplomfilm Haru Ichiban („Frühlingssturm“, fiktionaler Kurzfilm) wurde auf diversen internationalen Filmfestivals aufgeführt und beim Filmfestival Premier Plans in Angers mit dem Prix CinéCinéma ausgezeichnet. 2015 feierte ihr erster langer Kinodokumentarfilm Als die Sonne vom Himmel fiel Weltpremiere auf dem 68. Internationalen Filmfestival von Locarno (Semaine de la Critique). Der Film gewann den Schweizer Filmpreis 2016 für „Beste Filmmusik“ und war zudem in der Kategorie „Bester Dokumentarfilm“ nominiert. Seit Abschluss ihres Regiestudiums arbeitet Aya Domenig als Regisseurin, Cutterin, Filmproduzentin, Mentorin und wissenschaftliche Mitarbeiterin in interdisziplinären Forschungsprojekten.

Johanna Rolshoven

ist Kulturwissenschaftlerin und seit 2009 Professorin am Institut für Kulturanthropologie der Universität Graz (https://homepage.uni-graz.at/ de/johanna.rolshoven). Mit Schwerpunkten in der Stadtforschung, der politischen Anthropologie, den Mobile Culture Studies sowie den Culture Studies in Architecture erforscht sie global und postkolonial induzierte gesellschaftliche Transformationsprozesse in ihren Auswirkungen auf die dynamischen Räume, die sich zwischen Lebenswelten, ungleich strukturierter Gesellschaft und gebauter Umwelt aufspannen. Aktuelle Forschungsprojekte: Advancing the Value of the Humanities (www.valhuman.com), Rhythmanalysis of Mediterranean Port Cities Revisited (https://rhythmanalysis.iacsa.eu), Hirak. Ein Ohr voll Algerien in Bewegung (Radiofeature: Link zum Nachhören).

Jüngste Publikation:

Rolshoven, Johanna (2021): Stadt Raum Kulturanalyse.

Eine Einführung in die kulturanalytische Stadtforschung.

Bielefeld (Buchpublikation in Print)
Thomas Schärer

ist Historiker sowie promovierter Kultur- und Filmwissenschaftler. Er arbeitete als Journalist, als Kurator von Filmprogrammen (etwa in Locarno als Co-Leiter der Semaine de la Critique) und war Mitinitiant von Ausstellungen (unter anderem L'Histoire c'est moi, http://www.archimob. $\mathrm{ch} / \mathrm{f} /$ index.html). Zudem realisiert er Kurzdokumentarfilme und initiiert seit 2003 Forschungsprojekte (z. B. Cinémémoire: Eine Oral History des Schweizer Films, https://www.zhdk.ch/forschungsprojekt/cinememoireeine-oral-history-des-schweizer-films-426804). Seit 2009 lehrt Schärer an der Zürcher Hochschule der Künste (ZHdK) in den Departementen Kulturanalysen und Vermittlung sowie Design. Er publizierte zahlreiche kulturhistorische Artikel und filmgeschichtliche Monografien.

Jüngste Publikationen:

Minor Cinema. Experimental Film in Switzerland

(https://www.lespressesdureel.com/EN/ouvrage.php?id=7731),

Gifttod, Betonwüsten, strahlende Zukunft - Umweltbewegungen

und bewegte Bilder in der Schweiz, 1940-1990

(https://boris.unibe.ch/id/eprint/142141)

Julia Weber

ist Soziologin und Künstlerin. In ihren prozess- und forschungsbasierten Projekten, die sich an der Schnittstelle von Dokumentation und Fiktion, Ethnografie und Kunst ansiedeln lassen, geht sie gesellschaftlichen Paradoxien auf den Grund und experimentiert mit unterschiedlichen medialen Zugängen zur Alltagswirklichkeit (Fotografie, Text, Audio, Performance). Ihr besonderes Interesse gilt dem Wahrnehmen, Fühlen, Denken und Handeln von Menschen, welche in öffentlichen Diskursen mit eigener Stimme kaum repräsentiert sind. Aktuell verfolgt sie eine künstlerisch-ethnografische Promotion zum Thema „,Herumlungern“ als öffentliche Alltagspraxis - ,Herumlungern“ als künstlerische Strategie“ an der Kunstuniversität Linz. Außerdem konzipiert sie seit 2012 Community-Art-Workshops mit unterschiedlichen sozialen Gruppen.

Jüngste Publikation:

Weber, Julia (2018): „,Loitering‘ in Urban Public Space - Wandering with a Street Poet in Berlin“, in: WiderScreen: Kaupunkikuvitelmat ja urbaani arki - City imaginings and urban everyday life (1-2), S. 1-9 


\section{IMPRESSUM}

Band 25 der Schriftenreihe des Institute for Contemporary Art Research der Zürcher Hochschule der Künste

Die Publikation wurde durch den Schweizerischen Nationalfonds (SNF) finanziell unterstützt.

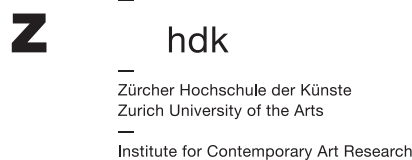

C 2021 Jürgen Krusche / Aya Domenig / Thomas Schärer / Julia Weber publiziert von der jovis Verlag $\mathrm{GmbH}$, Berlin

Fotocredits: Jürgen Krusche S. 1, 6, 14, 88 und 206

Lektorat: Katharina Freisinger

Gestaltung und Satz: Theresa Hattinger

Lithografie: Bild1Druck, Berlin

Gedruckt in der Europäischen Union

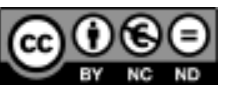

Dieses Werk ist lizensiert unter der

Creative-Commons-Lizenz CC BY-NC-ND 4.0.

Diese Lizenz erlaubt die Verbreitung

des Werks ausschließlich in unbearbeiteter Form und zu nichtkommerziellen Zwecken sowie unter Nennung der UrheberInnen.

(Lizenztext: https://creativecommons.org/licenses/by-nc-nd/4.0/)

Bibliografische Information der Deutschen Nationalbibliothek Die Deutsche Nationalbibliothek verzeichnet diese Publikation in der Deutschen Nationalbibliografie; detaillierte bibliografische Daten sind im Internet über http://dnb.d-nb.de abrufbar.

jovis Verlag $\mathrm{GmbH}$

Lützowstraße 33

10785 Berlin

www.jovis.de

jovis-Bücher sind weltweit im ausgewählten Buchhandel erhältlich. Informationen zu unserem internationalen Vertrieb erhalten Sie von Ihrem Buchhändler oder unter www.jovis.de.

ISBN 978-3-86859-643-4 (Softcover)

ISBN 978-3-86859-980-0 (PDF)

ISBN 978-3-86859-940-4 (Enriched E-Book)

DOI: https://doi.org/10.1515/978868599800
Unser Dank geht an alle InterviewpartnerInnen, ExpertInnen sowie all diejenigen, die uns an ihren Erfahrungen und ihrem Wissen teilhaben ließen und in dieser Publikation unerwähnt bleiben.

Für ihre Unterstützung vor Ort danken wir insbesondere Sigmar Gude von TOPOS, Berlin, den MitarbeiterInnen von Gangway e.V. - Verein für Straßensozialarbeit, dem Quartiersmanagement der DüttmannSiedlung sowie dem Getränkemarkt Hoffmann.

Unser Dank geht auch an Mirjam Gautschi, Mitarbeiterin im ersten Jahr des Projekts sowie an wichtige GesprächspartnerInnen: Johanna Rolshoven, Uriel Orlov, Elisabeth Bina Mohn, Franziska Nyffenegger und Mehdi Sahebi. 